\title{
Classificação de Esteatose Hepática Não Alcoólica em Imagens Térmicas da Região do Fígado Utilizando Redes Neurais Convolucionais
}

\author{
Daniel Moreira Pinto ${ }^{1}$, Johnatan Carvalho Souza ${ }^{1}$, Aristófanes Corrêa Silva ${ }^{1}$, \\ Henrique Manoel de Araujo Martins Filho ${ }^{2,3}$, Anselmo Cardoso de Paiva ${ }^{1}$, \\ Renato Amaro Zângaro ${ }^{2,4}$
}

${ }^{1}$ Núcleo de Computação Aplicada - Universidade Federal do Maranhão (UFMA) Av. dos Portugueses, 1966 - Vila Bacanga, São Luís - MA, 65080-805

${ }^{2}$ Universidade Anhembi Morumbi - UAM

Rua Casa do Ator, 294, São Paulo, SP, Brasil, 04546-001

${ }^{3}$ Alchimia

Rua Mar. Castelo Branco 31, Olho D’água, São Luís, MA, Brasil, 65065-090

${ }^{4}$ Centro de Inovação, Tecnologia e Educação - CITE

Parque Tecnológico de São José dos Campos,

Estrada Dr. Altino Bondensan, 500, São José dos Campos, SP, Brasil, 12247-016

\{daniel.pinto, johnatancarvalho\}@nca.ufma.br, ari@dee.ufma.br,

hmartinsfilho@globo.com, paiva@deinf.ufma.br, razangaro@gmail.com

\begin{abstract}
The non-alcoholic fatty liver disease (NAFLD) has the highest incidence among the liver diseases, afflicting about 1.5 billion people worldwide. It presents a high mortality rate and, if not dianosed and treated early, can evolve to severe liver complications. For this reason, it requires a fast and efficient diagnosis. Gauging of human body temperature with thermography is a noninvasive method of obtaining data that may indicate the presence of the disease. In this context, the purpose of this work is to present a method of NAFLD classification in thermography images of the liver. By using convolutional neural networks and image processing techniques, the proposed method achieved an accuracy of $96 \%$, sensitivity of $91 \%$ and specificity of $97 \%$.
\end{abstract}

Resumo. A esteatose hepática não alcoólica (DHGNA) é a doença de fígado de maior incidência no mundo, afetando cerca de 1,5 bilhão de pessoas. Ela possui uma alta taxa de mortalidade e, se não for diagnosticada e tratada cedo, pode evoluir para sérias complicações no fígado, portanto seu diagnóstico deve ser rápido e eficiente. A aferição da temperatura do corpo humano via termografia é um método não invasivo para detectar a DHGNA. Neste contexto, o objetivo deste trabalho é apresentar um método para classificação de DHGNA em imagens de termografia de fígado. Utilizando redes neurais convolucionais e técnicas de processamento de imagens, o método proposto foi capaz alcançar uma acurácia de 96\%, sensibilidade de $91 \%$ e especificidade $97 \%$. 


\section{Introdução}

A esteatose hepática não alcoólica ou doença hepática gordurosa não alcoólica (DHGNA) é caracterizada pela infiltração de gordura no fígado de indivíduos que não possuam um significativo histórico de abuso de álcool ou outras doenças hepáticas que expliquem essa infiltração, sendo em muitos casos associada com síndrome metabólica [Cotrim et al. 2016]. A DHGNA acompanha a obesidade e, globalmente, chega a afetar aproximadamente 1,5 bilhão de pessoas, o que faz dela a doença de fígado de maior alcance no mundo [Schwabe et al. 2020]. Em 25\% dos pacientes a doença evolui para esteato-hepatite não alcoólica, trazendo uma séria ameaça à vida desses pacientes com o aumento do risco do desenvolvimento de cirrose, falha do fígado e carcinoma hepatocelular [Schwabe et al. 2020].

Com o crescente número de casos da DHGNA [Schwabe et al. 2020] e, tendo em vista sua alta taxa de mortalidade [Cotrim et al. 2016], um diagnóstico rápido e eficiente é de grande importância para a saúde global. O exame mais específico para avaliar a presença e grau de desenvolvimento da DHGNA é hoje a biópsia hepática [Santana et al. 2021], porém esse método é invasivo e traz, além de custos altos, riscos à saúde do paciente submetido ao exame [Byra et al. 2018]. Portanto, a biópsia não é considerada o método mais otimizado para o diagnóstico da doença [Byra et al. 2018], o que torna o estudo de outros métodos extremamente importante, como os que utilizam ultrassonografia na região do abdômen [Byra et al. 2018] e termografia [Farooq and Corcoran 2020].

A termografia é um método não invasivo e que, ao contrário da ultrassonografia, não envolve contato físico para a extração de características corporais que podem ser utilizadas na avaliação da saúde de um paciente [Farooq and Corcoran 2020]. A temperatura do corpo humano é uma informação de extrema importância para o diagnóstico e monitoramento de doenças [Farooq and Corcoran 2020]. Em pacientes com DHGNA, observa-se que o desenvolvimento da doença causa a diminuição da temperatura superficial do fígado [Farooq and Corcoran 2020]. Portanto, a termografia é uma técnica não invasiva promissora para o diagnóstico de DHGNA [Brzezinski et al. 2020].

A qualidade da captura das emissões de infravermelho que câmeras térmicas utilizam para gerar os termogramas da superfície do corpo humano sofrem influência do ambiente, o que resulta na atenuação dos efeitos termográficos de anomalias corporais [Farooq and Corcoran 2020], dificultando sua detecção. Sendo assim, técnicas de melhoramento de imagem são úteis para reduzir ruídos e refinar características de imagens térmicas após sua coleta [Wahab et al. 2018]. Além disso, essas imagens podem ser introduzidas em modelos de aprendizagem de máquina para uma extração mais eficiente de informações que contribuam com a detecção de doenças [Farooq and Corcoran 2020].

Modelos de aprendizagem de máquina baseados em aprendizagem profunda têm mostrado excelente progresso em tarefas de classificação [Shorten and Khoshgoftaar 2019]. No ramo da aprendizagem profunda, foram desenvolvidas as redes neurais convolucionais (CNNs), que foram capazes de executar tarefas de visão computacional com grande sucesso [Shorten and Khoshgoftaar 2019]. As CNNs são hoje uma das principais técnicas de reconhecimento de padrões em imagens, inclusive imagens térmicas [Zuluaga-Gomez et al. 2020] e, na área clínica, têm sido extensamente utilizadas em tarefas de visão computacional [Shorten and Khoshgoftaar 2019]. 
Tendo em vista o grande impacto da DHGNA e a demanda de um diagnóstico eficiente e não invasivo, o presente trabalho tem como objetivo classificar termogramas da região do fígado como paciente saudável ou paciente com DHGNA, utilizando uma CNN. Ele está estruturado em 5 seções. A Seção 2 apresenta os trabalhos relacionados ao estudo. A Seção 3 apresenta a base de imagens utilizada no estudo e os métodos de coleta, pré-processamento e expansão da base, referenciando também a CNN testada. A Seção 4 apresenta os resultados obtidos. Por fim, a Seção 5 apresenta as conclusões do trabalho.

\section{Trabalhos Relacionados}

Nos últimos anos, descobertas sobre a utilização de termografia para o diagnóstico de DHGNA promovem o estudo de métodos que utilizem aprendizagem de máquina para diagnosticar a doença através das imagens geradas por essa técnica [Farooq and Corcoran 2020, Brzezinski et al. 2020]. Os principais métodos para o reconhecimento de padrões em imagens, inclusive imagens térmicas, são os que utilizam CNNs [Zuluaga-Gomez et al. 2020]. Esses métodos foram aplicados com sucesso para o diagnóstico de câncer de mama [Zuluaga-Gomez et al. 2020], chamando a atenção para o desenvolvimento de adaptações desses métodos para outras doenças detectáveis através de temperatura. Levando isso em consideração, nesta seção são apresentados os trabalhos utilizados como ponto de partida para o desenvolvimento do método proposto de diagnóstico de DHGNA a partir de termografia e utilizando CNNs.

O trabalho de Brzezinski et al. [Brzezinski et al. 2020] apresenta a primeira tentativa de uso de termografia da pele na região do fígado para o monitoramento in vivo de esteato-hepatite, estágio mais desenvolvido da DHGNA, em ratos de laboratório. A doença foi induzida via alimentação nos animais, que foram então sedados e tiveram sua temperatura coletada por uma câmera térmica. Um algoritmo foi desenvolvido para extrair dados dos termogramas gerados, na região do fígado dos animais, e então detectar a doença utilizando aprendizagem de máquina. O algoritmo criado foi capaz de, após os estágios iniciais do estudo, detectar a doença com sucesso em $100 \%$ dos testes. Os autores concluem que o desenvolvimento dessa técnica em humanos pode levar a avanços importantes na utilização de imagens térmicas no campo da detecção de doenças crônicas de fígado.

O estudo de Zuluaga-Gomez et al. [Zuluaga-Gomez et al. 2020] explorou o estado atual do diagnóstico de câncer de mama a partir de imagens térmicas da base DMR-IR [Silva et al. 2014] e que utilizassem aprendizagem de máquina. A partir desse estudo, os autores buscaram desenvolver um novo método de diagnóstico da doença, utilizando essa base de imagens. Os autores realizaram, então, uma série de experimentos com CNNs de referência, comparando suas capacidade de classificação do câncer. O resultado dos experimentos comprovou a eficiência do uso de CNNs para o diagnóstico de câncer de mama através de termografia. Além disso, foi observado que CNNs mais simples têm melhor desempenho com bases de dados mais limitadas como a DMR-IR. Os autores treinaram, então, uma série de CNNs customizadas e que executavam otimização automática de seus hiperparâmetros. Também foram feitos experimentos para avaliar a eficiência de aumento de dados no desempenho das CNNs treinadas, que apresentaram menos falsos negativos e menos falsos positivos ao terem essa técnica aplicada em seu treinamento. Como resultado, o trabalho gerou redes pouco profundas que alcançam uma acurácia de $92 \%$ e uma 
média harmônica entre precisão e sensibilidade de $92 \%$.

Brzezinski et al. [Brzezinski et al. 2020] comprovaram o potencial do uso de termografia da superfície da pele na região do fígado para monitorar a DHGNA de forma não invasiva em seres vivos. Porém, ainda não foram realizados grandes avanços na extensão de seu trabalho para seres humanos. Já Zuluaga-Gomez et al. [Zuluaga-Gomez et al. 2020] conseguiram estabelecer um método bem-sucedido de diagnóstico de câncer de mama em imagens térmicas de uma base limitada utilizando CNNs, desenvolvimento que ainda não foi obtido na detecção de DHGNA [Brzezinski et al. 2020].

Levando em conta a análise das descobertas, limitações e escopo dos trabalhos relacionados, este trabalho propõe um método para o diagnóstico de DHGNA em termogramas da região do fígado em abdômens humanos, utilizando as capacidades de detecção de padrões em imagem das CNNs. A base de imagens, descrita na Seção 3, apresenta limitações semelhantes às da base DMR-IR [Zuluaga-Gomez et al. 2020], portanto técnicas de aumento de dados precisaram ser aplicadas para aumentar a capacidade de generalização da CNN utilizada [Shorten and Khoshgoftaar 2019].

\section{Metodologia Proposta}

Nesta seção é apresentado o método de classificação de DHGNA utilizando a rede neural convolucional AlexNet. O método é composto de 5 etapas. A etapa 1 consiste da obtenção da base de imagens. Na etapa 2 foi coletada a região do fígado das imagens da base. $\mathrm{Na}$ etapa 3 as tabelas de temperatura geradas pela etapa 2 foram convertidas em imagens térmicas e então melhoradas. Na etapa 4 a base de imagens foi artificialmente expandida através de aumento de dados e a CNN AlexNet foi treinada com os dados expandidos. Na etapa 5 é feita a validação de resultados por meio da análise das métricas de avaliação. As etapas estão ilustradas na Figura 1.
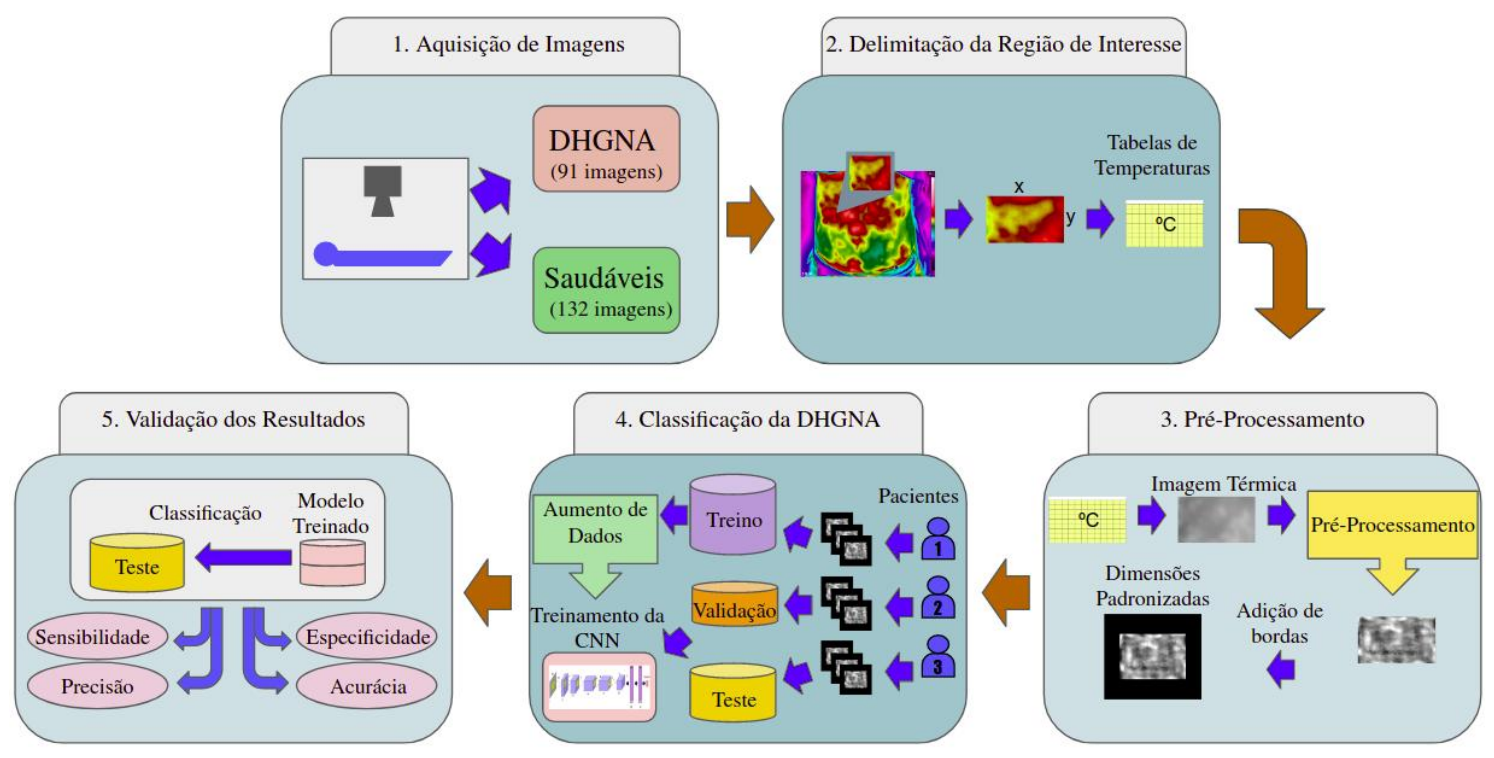

Figura 1. Etapas para a classificação de DHGNA 


\subsection{Aquisição de Imagens}

A base de imagens utilizada nos experimentos é constituída de 36 pacientes, estando 14 deles diagnosticados com DHGNA e 22 deles sem a doença. Cada paciente possui em média 6 imagens, totalizando 223 imagens, sendo 91 imagens de pacientes afetados pela doença e 132 imagens de pacientes não afetados. Relativamente, o grupo de pacientes com DHGNA possui mais imagens por paciente que o grupo sem a doença.

As imagens foram captadas no LIT - Laboratório de Imagem Térmica da clínica Alchimia, localizada na rua Marechal Castelo Branco, 31, Olho D’Água, São Luís - MA. Foi utilizado um termógrafo de sensor infravermelho FLIR - Modelo S650C de resolução 640x480, disponível comercialmente. Os pacientes foram aclimatizados por 15 minutos, em temperatura ambiente de $23^{\circ} \mathrm{C}$, com umidade relativa do ar de $65 \%$. A câmera foi posicionada à distância de 1,5 metro para a captura das imagens, estando o paciente na posição decúbito dorsal (deitado com o peito para cima) com a área abdominal desnuda durante toda a aclimatação e captação das imagens (Figura 2). Os pacientes foram diagnosticados como com DHGNA ou saudáveis através de ultrassonografia de abdômen superior ou abdômen total.

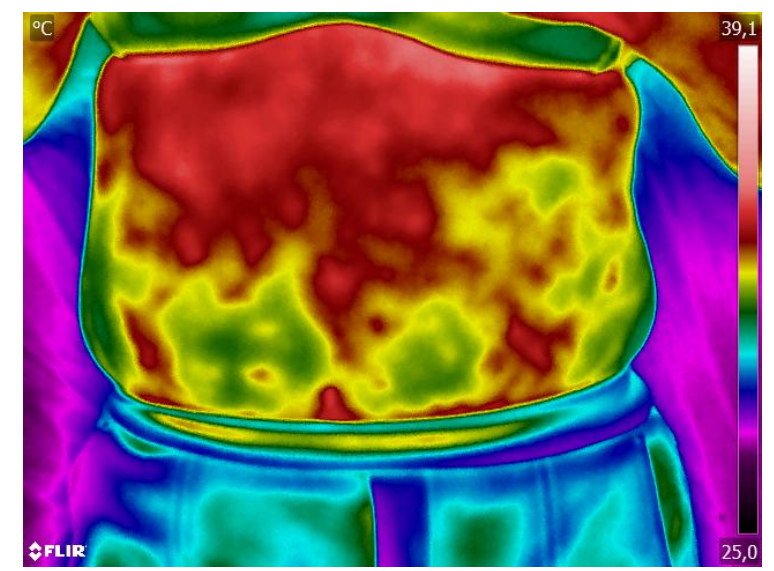

Figura 2. Termograma de paciente com DHGNA da base de imagens

\subsection{Delimitação da Região de Interesse}

A região de interesse (ROI) do abdômen dos pacientes é a região hepática (Figura 3), área anatômica onde o fígado está posicionado, no quadrante superior direito do abdômen [Ozougwu 2017, Brioschi et al. 2006]. As imagens da base possuem informações que não contribuem para o objetivo, como as regiões do abdômen fora da ROI, roupas, o plano de fundo, além de elementos extras como a barra de relação entre cores e temperaturas (Figura 2). Portanto, foi necessária a extração da ROI de cada imagem. Como as CNNs recebem como entrada uma sequência de imagens quadradas de mesma dimensão, optou-se por extrair a ROI sempre em formato retangular. Por causa das diferenças físicas entre os pacientes, ROIs de dimensões diferentes foram geradas. Também foram extraídas ROIs de dimensões quadradas e iguais para todos os pacientes, que aproximavam a região hepática de todos, para verificar se a CNN teria melhor desempenho com dados específicos ou dados padronizados. Porém, a rede teve mais dificuldade em aprender nos testes feitos com as imagens de ROIs padronizadas. 
As ROIs de cada imagem foram extraídas pelos autores por meio do software especializado FLIR Tools ${ }^{1}$. Com a orientação de um especialista, foi selecionada a região retangular mais adequada aos parâmetros da região hepática do paciente. As temperaturas da área foram exportadas como tabelas de temperaturas, onde cada célula contém o valor referente à temperatura, em graus Celsius, e com três casas decimais de precisão, de um pixel da imagem. Para cada paciente as ROIs extraídas de suas imagens possuem as mesmas dimensões, com diferenças de valores internos causadas pelo posicionamento manual da área retangular e pelo movimento do paciente durante a coleta das fotos.

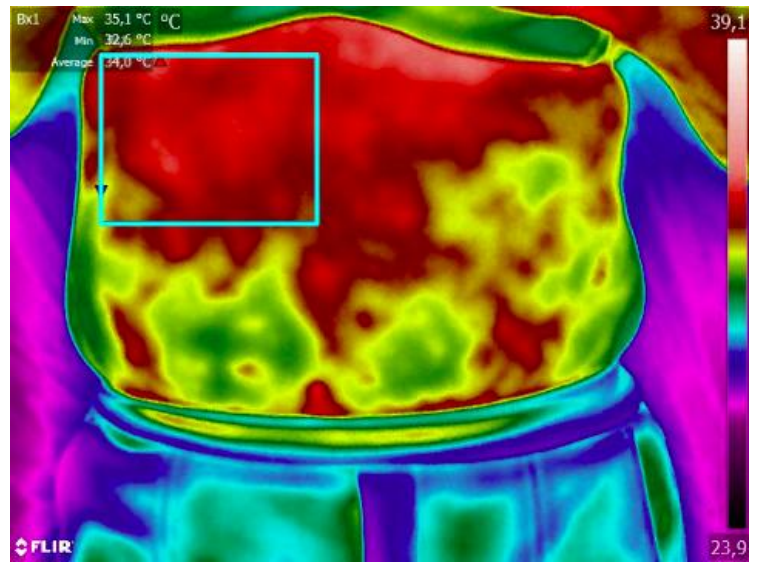

Figura 3. Termograma com região de interesse delimitada

\subsection{Pré-Processamento}

Como as ROIs são exportadas através do FLIR Tools como tabelas de temperaturas, foi necessário um passo de conversão das tabelas para imagens térmicas. As tabelas foram convertidas para imagens em escala de cinza (Figura 4), que foram então melhoradas com técnicas de pré-processamento já reportadas como eficientes no melhoramento de imagens térmicas [Wahab et al. 2018, Zuluaga-Gomez et al. 2020]. Dessa forma, foram realçados ao máximo os elementos visuais, como texturas e intensidades de pixel relacionadas à temperatura representada, além de ser feita a limpeza de ruídos gerados no momento da coleta da base. Além disso, nesse passo as ROIs tiveram suas dimensões padronizadas.

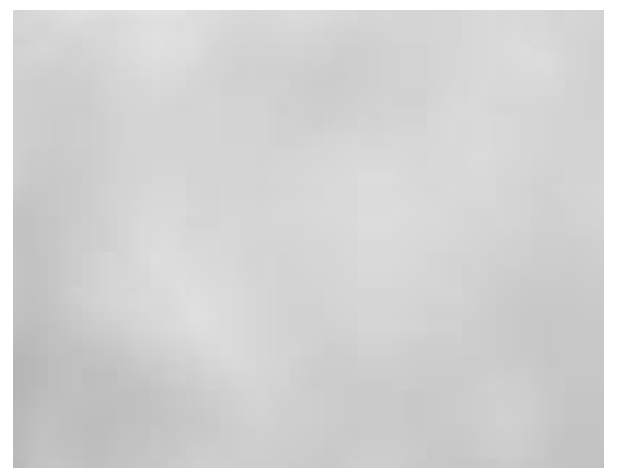

Figura 4. ROI convertida para imagem térmica

\footnotetext{
${ }^{1}$ Disponível em: https://www.flir.com.br/products/flir-tools/
} 
A conversão das ROIs para imagens térmicas foi realizada através de uma função de conversão linear representada pela Equação 1

$$
f(t)=\frac{(t * 1000-\text { oldMin }) *(\text { newMax }- \text { newMin })}{(\text { oldMax }- \text { oldMin })}+\text { newMin }
$$

onde $t$ é a temperatura de uma célula da tabela, oldMin é a temperatura mínima entre todas as ROIs da base, multiplicada por 1000, oldMax é a temperatura máxima entre todas as ROIs da base, multiplicada por 1000, new Min é o valor mínimo de um pixel (0) e newMax é o valor máximo de um pixel (255). Dessa forma, cada valor de temperatura foi convertido em uma intensidade de cinza, preservando completamente a precisão de detalhes até a primeira posição decimal de temperatura e parcialmente até a segunda posição decimal. Utilizar mínimo e máximo universais de temperatura, ou seja, a menor temperatura entre todos os pacientes e a maior temperatura entre todos os pacientes, provou-se, após experimentos, ser muito mais eficiente que utilizar mínimo e máximo locais, onde oldMin e oldMax levam em conta a menor temperatura e a maior temperatura específicas a cada paciente dentre as suas imagens. A rede alcançou resultados de aprendizado no primeiro caso e mostrou uma extrema dificuldade em aprender no segundo caso.

Para o pré-processamento, foram experimentadas combinações de remoção de ruído e equalização de histograma vistos como úteis no melhoramento de imagens térmicas [Wahab et al. 2018], tendo sido bem-sucedida a combinação da remoção de ruído de wiener [Wahab et al. 2018] e equalização de histograma adaptativa (CLAHE) [Zuluaga-Gomez et al. 2020]. Além disso, para que pudessem ser inseridas na CNN, as ROIs receberam bordas de pixels pretos que padronizaram as imagens resultantes com lados iguais, medindo o mesmo que a maior dimensão dentre todas as imagens, o que resultou em imagens de $283 \times 283$ pixels, ficando a região centralizada na imagem (Figura 5).
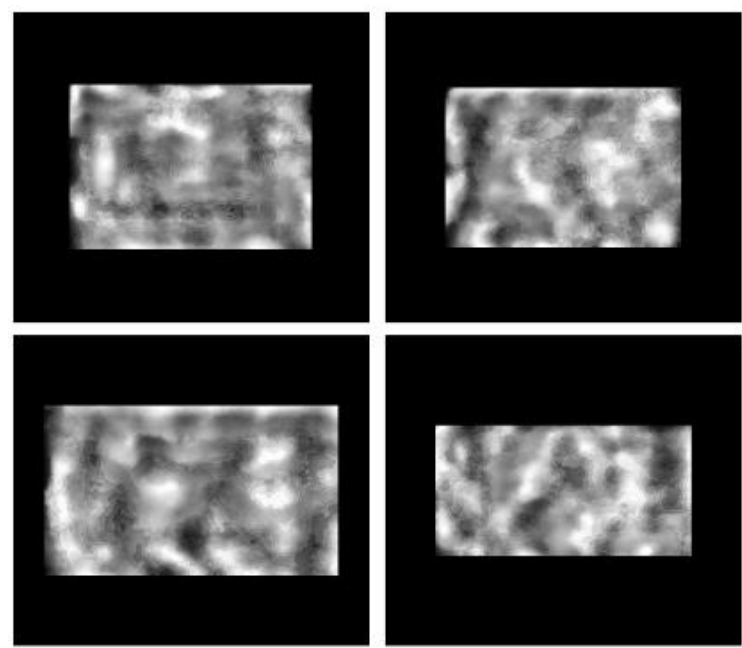

Figura 5. ROls de diferentes pacientes pré-processadas e com borda

\subsection{Classificação da DHGNA}

Nesta etapa é realizada a classificação da DHGNA utilizando a CNN AlexNet. A base detalhada na Seção 3.1 foi dividida em conjuntos de treino, validação e teste, de forma balanceada entre as classes. O conjunto de treino passou, então, por um processo de aumento 
de dados, que aplicou transformações em suas imagens para gerar imagens sintéticas, expandindo o conjunto em duas vezes o seu tamanho. Finalmente, a AlexNet treinou com o conjunto de treino aumentado, realizando validação com o conjunto de validação a cada época durante o treino. Os resultados obtidos na predição, sobre o conjunto de teste, do modelo treinado são apresentados na Seção 4.

Para a divisão dos conjuntos, os pacientes foram divididos entre conjuntos de treino, validação e teste, de forma que não houvessem imagens de um mesmo paciente em conjuntos diferentes [Zuluaga-Gomez et al. 2020], para evitar considerar resultados de modelos possivelmente ineficientes em generalização (apresentando overfitting). Os pacientes com DHGNA estão em menor número na base, portanto foi necessário balancear o conjunto de treino entre as duas classes. A divisão iniciou com a separação de uma partição de 20\% dos pacientes com DHGNA para o conjunto de teste [Géron 2019, p. 31] e $20 \%$ para o conjunto de validação durante o treinamento. Os $60 \%$ restantes dos pacientes com a doença foram incluídos no conjunto de treino. Em seguida, foram adicionados pacientes sem a doença no conjunto de treino, em número idêntico aos pacientes com DHGNA no conjunto. O mesmo processo foi feito no conjunto de validação e o restante dos pacientes sem DHGNA foi incluído no conjunto de teste. O conjunto de treino possuiu 49 imagens com DHGNA e 43 imagens sem a doença. O conjunto de validação possuiu 19 imagens com DHGNA e 18 imagens sem a doença. Por fim, o conjunto de teste possuiu 23 imagens com DHGNA e 71 imagens sem a doença.

A pouca quantidade de imagens e o desbalanceamento da base configura um usual problema das CNNs aplicadas a tarefas de visão computacional na área clínica [Shorten and Khoshgoftaar 2019], portanto, foi necessária a aplicação de aumento de dados no conjunto de treino. Transformações foram aplicadas às imagens de treino para gerar novas imagens sintéticas, expandindo o conjunto. Foram testados vários níveis de expansão, para buscar os mais eficientes. A expansão máxima suportada foi de 100 vezes a quantidade de imagens do conjunto de treino. O nível de expansão mais eficiente identificado foi o de duas vezes a quantidade de imagens do conjunto de treino. As transformações utilizadas foram giros horizontais e verticais, zoom de até $20 \%$ e adição de ruído gaussiano [Shorten and Khoshgoftaar 2019].

Finalmente, as imagens foram utilizadas para treinamento da CNN AlexNet [Krizhevsky et al. 2012], experimentando trazer para o diagnóstico de DHGNA as conclusões de Zuluaga-Gomez et al. [Zuluaga-Gomez et al. 2020] sobre o melhor desempenho de CNNs mais simples sobre bases mais limitadas de imagens térmicas. A rede possui cinco camadas de convolução, três camadas de pooling e três camadas completamente conectadas, sendo a rede inicializada com pesos aleatórios. Para o método, foi utilizada a função de ativação sigmoide, função de perda binary cross-entropy, otimizador Adam e tamanho de batch de 32 imagens. A rede treinou por 100 épocas. O esquema da AlexNet utilizada está representado na Figura 6. A rede foi treinada em uma GPU NVIDIA Tesla K80, disponível gratuitamente através do Google Colab.

\subsection{Validação dos Resultados}

Para a classificação, pacientes com DHGNA foram considerados como a classe positiva e os saudáveis como a classe negativa. O desempenho dos modelos foi avaliado a partir de quatro métricas, sendo essas a precisão, sensibilidade, especificidade e acurácia. Cada 


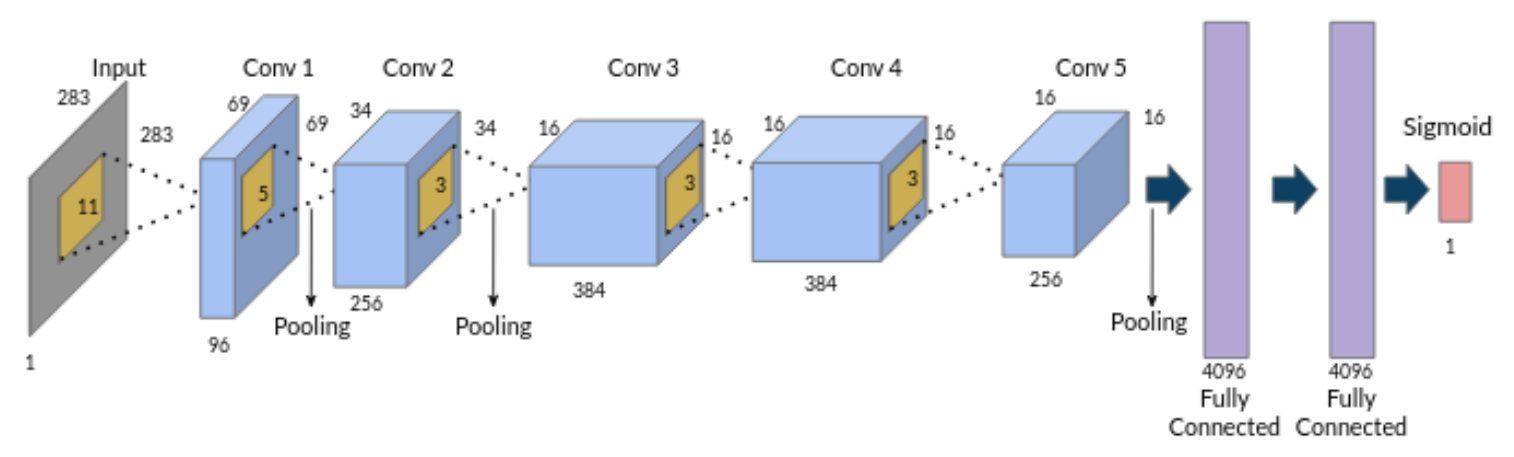

Figura 6. Representação da AlexNet utilizada

uma das métricas é apresentada nas equações a seguir, sendo $V P$ os verdadeiros positivos, imagens corretamente classificadas com a doença, $F P$ os falsos positivos, imagens incorretamente classificadas com a doença, $V N$ os verdadeiros negativos, imagens corretamente classificadas sem a doença e $F N$ os falsos negativos, imagens incorretamente classificadas sem a doença.

$$
\begin{gathered}
\text { precisão }=\frac{V P}{V P+F P} \\
\text { sensibilidade }=\frac{V P}{V P+F N} \\
\text { especificidade }=\frac{V N}{V N+F P} \\
\text { acurácia }=\frac{V P+V N}{V P+F P+V N+F N}
\end{gathered}
$$

A precisão (Equação 2) verifica, dentre as classificações de casos com a doença realizadas pelo modelo, quantas estão corretas. A sensibilidade (Equação 3) é a proporção de verdadeiros positivos, ou seja, a capacidade do modelo em identificar corretamente a doença em casos que realmente a têm. A especificidade (Equação 4) é a proporção de verdadeiros negativos, ou seja, a capacidade do modelo em indicar corretamente a ausência da doença em casos que realmente não a têm. A acurácia (Equação 5) é a proporção de acerto da classificação no geral, sem levar em conta as classes.

\section{Resultados}

Nesta seção, são apresentados os resultados do método proposto de classificação de DHGNA utilizando termografia e uma CNN. Inicialmente são discutidas as métricas utilizadas para avaliar o desempenho da CNN utilizada, então são apresentados os melhores resultados alcançados. Adicionalmente, é apresentado o que esse método estende das descobertas dos trabalhos relacionados.

Em aplicações clínicas, o risco maior à vida dos pacientes encontra-se na não detecção da doença em pacientes doentes [Zuluaga-Gomez et al. 2020]. Como a sensibilidade, representada na Equação 3, penaliza a classificação de falsos negativos, essa métrica torna-se a mais importante na avaliação dos modelos. Falsos negativos devem ser evitados ao máximo para aumentar a chance de um diagnóstico eficiente de pacientes doentes, diminuindo a mortalidade da doença [Zuluaga-Gomez et al. 2020]. Portanto, os 
modelos foram avaliados em primeiro lugar a partir da sensibilidade que alcançaram e, em segundo lugar, pela precisão (Equação 2), que, por sua vez, permite evitar modelos que classifiquem muitos falsos positivos.

Os resultados alcançados mostram que a AlexNet possui boa capacidade de classificar entre as imagens térmicas das regiões hepáticas afetadas por DHGNA e as saudáveis. Com a aplicação do método, foi alcançada uma sensibilidade de $91 \%$, precisão de $91 \%$, especificidade de $97 \%$ e acurácia de $96 \%$. Desses resultados, pode-se observar que a utilização de termografias da superfície da pele sobre a região do fígado para diagnóstico de DHGNA, com base em aprendizagem de máquina, foi estendida com resultados promissores para seres humanos. Além disso, foi mostrado que a AlexNet é uma CNN eficiente no reconhecimento de padrões que podem ser associados à presença da doença, e que as técnicas atualmente bem-sucedidas de aplicação de redes como essa em tarefas clínicas podem ser trazidas à tarefa de detecção da DHGNA.

\section{Conclusão}

Este trabalho aplicou técnicas de aprendizagem de máquina através de uma rede neural convolucional para classificação de esteatose hepática não alcoólica usando imagens térmicas da região do fígado. Através do método construído, a AlexNet utilizada foi capaz de alcançar alta sensibilidade e métricas gerais promissoras para a sua aplicação no diagnóstico de DHGNA, mesmo com uma base limitada. Com o método, foi visto que a seleção da região hepática adequada de cada paciente cria uma base de dados que torna o treinamento da CNN mais eficiente, ao contrário do uso de uma ROI padronizada. Além disso, foram verificadas as técnicas de melhoramento de imagem e aumento de dados que mais melhoram o aprendizado da CNN.

O método proposto precisou ser desenvolvido a partir das descobertas de trabalhos cujo escopo atende de forma limitada o problema abordado nesse trabalho. Portanto, mais estudos sobre a utilização de CNNs para a deteção da DHGNA a partir de termografia precisam ser realizados, para otimizar as técnicas de aplicação das redes. Para trabalhos futuros, propõe-se experimentar técnicas adicionais da utilização de CNNs, como a aplicação de redes pré-treinadas e redes de otimização automática, bem como experimentar outras CNNs de referência. Um estudo da melhor representação da região hepática também vê-se necessário, para definir a melhor entrada para o treinamento das redes.

\section{Agradecimentos}

Os autores agradecem ao Conselho Nacional de Desenvolvimento Científico e Tecnológico pelo financiamento do trabalho, e à Universidade Federal do Maranhão pelo apoio fornecido.

\section{Referências}

Brioschi, M. L., Yeng, L. T., Colman, D., Silva, F. M. M., and Teixeira, M. J. (2006). Imaginologia infravermelha no estudo avançado da dor de origem visceral* infrared imaging in the advanced study of visceral pain.

Brzezinski, R. Y., Levin-Kotler, L., Rabin, N., Ovadia-Blechman, Z., Zimmer, Y., Sternfeld, A., Finchelman, J. M., Unis, R., Lewis, N., Tepper-Shaihov, O., et al. (2020). 
Automated thermal imaging for the detection of fatty liver disease. Scientific reports, 10(1):1-11.

Byra, M., Styczynski, G., Szmigielski, C., Kalinowski, P., Michałowski, Ł., Paluszkiewicz, R., Ziarkiewicz-Wróblewska, B., Zieniewicz, K., Sobieraj, P., and Nowicki, A. (2018). Transfer learning with deep convolutional neural network for liver steatosis assessment in ultrasound images. International journal of computer assisted radiology and surgery, 13(12):1895-1903.

Cotrim, H. P., Parise, E. R., Figueiredo-Mendes, C., Galizzi-Filho, J., Porta, G., and Oliveira, C. P. (2016). Nonalcoholic fatty liver disease brazilian society of hepatology consensus. Arquivos de gastroenterologia, 53(2):118-122.

Farooq, M. A. and Corcoran, P. (2020). Infrared imaging for human thermography and breast tumor classification using thermal images. In 2020 31st Irish Signals and Systems Conference (ISSC), pages 1-6. IEEE.

Géron, A. (2019). Hands-on machine learning with Scikit-Learn, Keras, and TensorFlow: Concepts, tools, and techniques to build intelligent systems. O'Reilly Media.

Krizhevsky, A., Sutskever, I., and Hinton, G. E. (2012). Imagenet classification with deep convolutional neural networks. Advances in neural information processing systems, 25:1097-1105.

Ozougwu, J. C. (2017). Physiology of the liver. International Journal of Research in Pharmacy and Biosciences, 4(8):13-24.

Santana, J. T., Mota, A. V. H., Gonzaga, Y. H. G., Gomes, R. M. O. P., Melo, L. C., Noronha, V. F. C. M., Santos, A. C. O. L., de Jesus, J. B., Lima, S. O., and Cruz, J. F. (2021). Perfil metabólico e antropométrico dos pacientes obesos e não obesos portadores de esteatose hepática não alcoólica. Revista Eletrônica Acervo Saúde, 13(2):e5525-e5525.

Schwabe, R. F., Tabas, I., and Pajvani, U. B. (2020). Mechanisms of fibrosis development in nonalcoholic steatohepatitis. Gastroenterology, 158(7):1913-1928.

Shorten, C. and Khoshgoftaar, T. M. (2019). A survey on image data augmentation for deep learning. Journal of Big Data, 6(1):1-48.

Silva, L., Saade, D., Sequeiros, G., Silva, A., Paiva, A., Bravo, R., and Conci, A. (2014). A new database for breast research with infrared image. Journal of Medical Imaging and Health Informatics, 4(1):92-100.

Wahab, A. A., Salim, M., Yunus, J., and Ramlee, M. H. (2018). Comparative evaluation of medical thermal image enhancement techniques for breast cancer detection. Journal of Engineering and Technological Sciences, 50:40-52.

Zuluaga-Gomez, J., Al Masry, Z., Benaggoune, K., Meraghni, S., and Zerhouni, N. (2020). A cnn-based methodology for breast cancer diagnosis using thermal images. Computer Methods in Biomechanics and Biomedical Engineering: Imaging \& Visualization, pages 1-15. 